\title{
Multiple Solutions for Asymptotically Linear Elliptic Systems
}

Fukun Zhao, Leiga Zhao and Yanheng Ding

Abstract. This paper is concerned with the following periodic Hamiltonian elliptic system

$$
\left\{\begin{array}{lll}
-\Delta u+V(x) u=g(x, v) & \text { in } \quad \mathbb{R}^{N}, \\
-\Delta v+V(x) v=f(x, u) & \text { in } \quad \mathbb{R}^{N}, \\
u(x) \rightarrow 0 \text { and } v(x) \rightarrow 0 & \text { as } \quad|x| \rightarrow \infty,
\end{array}\right.
$$

where the potential $V$ is periodic and has a positive bound from below, $f(x, t)$ and $g(x, t)$ are periodic in $x$, asymptotically linear in $t$ as $|t| \rightarrow \infty$. By using critical point theory of strongly indefinite functionals, existence of a positive ground state solution as well as infinitely many geometrically distinct solutions for odd $f$ and $g$ are obtained.

Mathematics Subject Classification (2000). Primary 35J50; Secondary 35J55. Keywords. Hamiltonian elliptic system, variational method, strongly indefinite functional.

\section{Introduction and main results}

In this paper, we study nonlinear elliptic systems in Hamiltonian form

$$
\begin{cases}-\Delta u+V(x) u=g(x, v) & \text { in } \quad \mathbb{R}^{N}, \\ -\Delta v+V(x) v=f(x, u) & \text { in } \quad \mathbb{R}^{N}, \\ u(x) \rightarrow 0 \text { and } v(x) \rightarrow 0 & \text { as } \quad|x| \rightarrow \infty,\end{cases}
$$

where $N \geq 1, V \in C\left(\mathbb{R}^{N}, \mathbb{R}\right)$ and $f, g \in C\left(\mathbb{R}^{N} \times \mathbb{R}, \mathbb{R}\right)$.

For the case of a bounded domain these systems were studied by a number of authors, for instance, Benci and Rabinowitz [8], de Figueiredo and Ding [11], de Figueiredo and Felmer [12], Hulshof and Van de Vorst [16], Kryszewski and

This work was supported partly by NSFC (10561011 and 10671195), NSFC of Yunnan Proviance, and the Foundation of Education Commission of Yunnan Province, China. 
Szulkin [18]. Recently, de Figueiredo, do Ó and Ruf [13] treated an autonomous system via an Orlicz space approach.

The problem (ES) in the whole space $\mathbb{R}^{N}$ was considered recently in some works. Most of them focused on the case $V \equiv 1$. The main difficulty of such type problem is the lack of the compactness of the Sobolev embedding. An usual way to overcome this difficulty is that of working in the space of radially symmetric functions, where one recovers a compact embedding. By this means, de Figueiredo and Yang [14] obtained a positive radially symmetric solution which decay exponentially to zero at infinity. Their result were generalized later by Sirakov [25] in a different way. In their paper [6], Bartsch and de Figueiredo proved that the system admits infinitely many radial as well as non-radial solutions by the fountain theorem. Li and Yang [21] showed that, via a generalized linking theorem, (ES) has a positive ground state for asymptotically autonomous linear nonlinearities. Starting from this result they also proved existence of a positive solution for (ES) if $f(x, u)$ and $g(x, v)$ have autonomous limits $\bar{f}(t)$ and $\bar{g}(t)$ at infinity.

To avoid the indefinite character of the original functional, some authors used a dual variational method. See, for example, Ávila and Yang [4,5], Alves, Carrião, Miyagaki [3], Yang [28] and the references therein. See also Pistoia and Ramos [22] and the references therein for singularly perturbed problems.

In this paper we consider periodic asymptotically linear elliptic systems. As far as we know, there is no multiplicity result for such systems. Our purpose is to obtain an existence and, under stronger assumptions, a multiplicity result. Since there is no radial assumption we have to work on $H^{1}\left(\mathbb{R}^{N}\right) \times H^{1}\left(\mathbb{R}^{N}\right)$. A main difficulty when dealing with asymptotically linear problem is the lack of a priori bounds for the Palais-Smale or Cerami sequences of the associated functional. To overcome this difficulty we follow the approach originally developed in Jeanjean [17]. Namely assuming that such a sequence is unbounded we reach a contradiction by showing that neither the vanishing nor the non-vanishing can occur for the normalized sequence (see Lemma 4.1). Another main feature of such system is that the associated variational functional is strongly indefinite and its gradient is not of the form "identity minus compact". Therefore the generalized mountain pass theorem does not apply. However thanks to the periodic assumption, we can prove a weak version of the PS condition due to Coti Zelati and Rabinowitz [9] and [10]. This suffices to obtain the multiplicity result via the critical point theory of strongly indefinite functional which was developed recently by Bartsch and Ding [7].

On the potential $V$, we assume

$\left(\mathrm{V}_{0}\right) V \in C\left(\mathbb{R}^{N}, \mathbb{R}\right)$ is 1-periodic in each $x_{i}$ for $i=1, \ldots, N$ and $V_{0}:=\min V>0$.

Let $S:=-\Delta+V$ be the Schrödinger operator. Denote by $\sigma(S)$ and $\sigma_{\text {cont }}(S)$ the spectrum and the continuous spectrum of the operator $S$. It is well known that, under $\left(V_{0}\right)$, the spectrum $\sigma(S)=\sigma_{\text {cont }}(S) \subset\left[V_{0}, \infty\right)$ is a union of closed intervals (see Reed and Simon [23]). Next, we denote by $F(x, t)$ and $G(x, t)$ the primitives of $f(x, t)$ and $g(x, t)$, respectively. On the nonlinearities, we assume 
$\left(\mathrm{H}_{0}\right) f, g \in C\left(\mathbb{R}^{N} \times \mathbb{R}^{2}, \mathbb{R}\right)$ are 1-periodic in each $x_{i}$ for $i=1, \ldots, N$;

$\left(\mathrm{H}_{1}\right) \quad f(x, t)=o(|t|)$ and $g(x, t)=o(|t|)$ as $|t| \rightarrow 0$ uniformly in $x, t f(x, t), \operatorname{tg}(x, t)$ are both positive for all $(x, t)$ with $t \neq 0$;

$\left(\mathrm{H}_{2}\right) \lim _{t \rightarrow \infty} f(x, t) / t=l, \lim _{t \rightarrow \infty} g(x, t) / t=m$ uniformly in $x$;

$\left(\mathrm{H}_{3}\right) \min \{l, m\}>\max V\left(\mathbb{R}^{N}\right)$;

$\left(\mathrm{H}_{4}\right) \hat{F}(x, t) \geq 0, \hat{G}(x, t) \geq 0$, and there is a $\delta>0$ such that $\hat{F}(x, t)>0$ or $\hat{G}(x, t)>0$ if $0<|t| \leq \delta$, where $\hat{F}(x, t)=\frac{1}{2} f(x, t) t-F(x, t)$ and $\hat{G}(x, t)=$ $\frac{1}{2} g(x, t) t-G(x, t) ;$

$\left(\mathrm{H}_{5}\right)$ there exists $\delta_{0} \in\left(0, V_{0}\right)$ such that $\hat{F}(x, t) \geq \delta_{0}$ whenever $|f(x, t)| \geq t\left(V_{0}-\delta_{0}\right)$ and $\hat{G}(x, t) \geq \delta_{0}$ whenever $|g(x, t)| \geq t\left(V_{0}-\delta_{0}\right)$.

Observe that, due to the periodicity of $V, f$ and $g$, if $(u, v)$ is a solution of (ES), then so is $(a * u, a * v)$ for each $a \in \mathbb{Z}^{N}$, where $(a * u)(x)=u(x+a)$. Two solutions $\left(u_{1}, v_{1}\right)$ and $\left(u_{2}, v_{2}\right)$ are said to be geometrically distinct if $a * u_{1} \neq u_{2}$ or $a * v_{1} \neq v_{2}$ for all $a \in \mathbb{Z}^{N}$. Our main result is the following:

Theorem 1.1. Let $\left(\mathrm{V}_{0}\right)$ and $\left(\mathrm{H}_{0}\right)-\left(\mathrm{H}_{5}\right)$ be satisfied. Then (ES) has a positive ground state solution. If additionally $f(x, t)$ and $g(x, t)$ are odd in $t$, then (ES) has infinitely many geometrically distinct solutions.

Remark 1.2. $\left(\mathrm{H}_{3}\right)$ can be weakened to

$$
\left(\mathrm{H}_{3}^{\prime}\right) \min \{l, m\}>\inf \sigma(S) .
$$

Remark 1.3. In the case of $V \equiv 1$ and $f(x, t)$ and $g(x, t)$ are independent of $x$, the positive ground state solution was obtained in Theorem 1.1 of $\mathrm{Li}$ and Yang [21] under the assumptions similar to $\left(H_{0}\right)-\left(H_{3}\right),\left(H_{5}\right)$ and the following

$\left(\mathrm{H}_{4}^{\prime}\right) \hat{F}(t), \hat{G}(t) \geq 0$ and $\hat{F}(t), \hat{G}(t)>0$ if $t>0$.

$\left(\mathrm{H}_{6}\right) f(t) / t$ and $g(t) / t$ are non-decreasing in $t \geq 0$.

The paper is organized as follows. In Section 2, we set up the framework in which we study the variational problem associated to (ES). In Section 3, we discuss the linking structure of the functional. In Section 4, we show that the variational functional satisfies a weak version of $(C)_{c}$-condition. The proof of Theorem 1.1 announced above will be given in the last section.

\section{Variational setting}

Below by $|\cdot|_{q}$ we denote the usual $L^{q}$-norm, $c$ or $c_{i}$ stands for different positive constants. Let $X$ and $Y$ are two Banach spaces with norms $\|\cdot\|_{X}$ and $\|\cdot\|_{Y}$, we always choose the equivalent norm $\|(x, y)\|_{X \times Y}=\left(\|x\|_{X}^{2}+\|y\|_{Y}^{2}\right)^{1 / 2}$ on the product space $X \times Y$. In particular, if $X$ and $Y$ are two Hilbert spaces with inner products $(\cdot, \cdot)_{X}$ and $(\cdot, \cdot)_{Y}$, we choose the inner product $((x, y),(w, z))_{X \times Y}=$ $(x, w)_{X}+(y, z)_{Y}$ on the product space $X \times Y$. 
Under $\left(V_{0}\right),\|u\|_{V}=\left(\int_{\mathbb{R}^{N}}|\nabla u|^{2}+V(x) u^{2}\right)^{1 / 2}$ is an equivalent norm with the usual one on $H^{1}\left(\mathbb{R}^{N}\right)$. Let $E=H^{1}\left(\mathbb{R}^{N}\right) \times H^{1}\left(\mathbb{R}^{N}\right)$ with the inner product

$$
((u, v),(\varphi, \psi))=\int_{\mathbb{R}^{N}}(\nabla u \nabla \varphi+V(x) u \varphi+\nabla v \nabla \psi+V(x) v \psi)
$$

and the corresponding norm

$$
\|(u, v)\|=\left[\int_{\mathbb{R}^{N}}\left(|\nabla u|^{2}+V(x) u^{2}+|\nabla v|^{2}+V(x) v^{2}\right)\right]^{1 / 2} .
$$

The system (ES) is naturally variational and its energy functional is

$$
\Phi(z)=\Phi(u, v)=\int_{\times \mathbb{R}^{N}} \nabla u \nabla v+V(x) u v-\Psi(z),
$$

for $z=(u, v) \in E$, where $\Psi(z)=\Psi(u, v)=\int_{\mathbb{R}^{N}} F(x, u)+G(x, v)$. Our hypotheses imply that $\Phi \in C^{1}(E, \mathbb{R})$. Moreover, a standard argument shows that the critical points of $\Phi$ are solutions of (ES).

Since

$$
\Phi^{\prime}(z) \eta=\Phi^{\prime}(u, v)(\varphi, \psi)=\int_{\mathbb{R}^{N}} \nabla u \nabla \psi+V(x) u \psi+\nabla v \nabla \varphi+V(x) v \varphi+\Psi^{\prime}(z) \eta,
$$

following De Figueiredo and Felmer [12], Hulshof and Van Der Vorst [16] (see also Figueiredo and Yang [14], Li and Yang [21]), we introduce a bilinear form

$$
B(z, \eta)=B((u, v),(\varphi, \psi))=\int_{\mathbb{R}^{N}} \nabla u \nabla \psi+V(x) u \psi+\nabla v \nabla \varphi+V(x) v \varphi
$$

and set

$$
E^{+}:=\left\{(u, u) \mid u \in H^{1}\left(\mathbb{R}^{N}\right)\right\} \quad \text { and } \quad E^{-}:=\left\{(u,-u) \mid u \in H^{1}\left(\mathbb{R}^{N}\right)\right\} .
$$

For each $z=(u, v) \in E$, let $z^{+}=((u+v) / 2,(u+v) / 2)$ and $z^{-}=((u-v) / 2,(v-$ $u) / 2)$, then $\left(z^{+}, z^{-}\right)=0$ and $\left(z^{+}, z^{-}\right)_{L^{2} \times L^{2}}=0$. Hence $E=E^{+} \oplus E^{-}$and

$$
\int_{\mathbb{R}^{N}} \nabla u \nabla v+V(x) u v=\frac{1}{2} B[z, z]=\frac{1}{2}\left(\left\|z^{+}\right\|^{2}-\left\|z^{-}\right\|^{2}\right) .
$$

Therefore, the functional $\Phi$ can be rewritten in a standard way

$$
\Phi(z)=\frac{1}{2}\left(\left\|z^{+}\right\|^{2}-\left\|z^{-}\right\|^{2}\right)-\Psi(z), \quad \text { for } \quad z=(u, v) \in E .
$$

The functional $\Phi$ is strongly indefinite, such type functional appeared extensively when one study differential equations via critical point theory, see for example [19, $26,27]$ and the references therein. 


\section{Linking structure}

In the sequel, we write $|z|=\left(|u|^{2}+|v|^{2}\right)^{1 / 2}$ for $z=(u, v)$. In this section, we discuss the linking structure of $\Phi$.

Lemma 3.1. Suppose $\left(\mathrm{H}_{0}\right)-\left(\mathrm{H}_{2}\right)$ are satisfied. Then there is a $\rho>0$ such that $\kappa:=\inf \Phi\left(\partial B_{\rho} \cap E^{+}\right)>0$.

Proof. Observe that, given $\varepsilon>0$, there is $C_{\varepsilon}>0$ such that

$$
|f(x, t)|,|g(x, t)| \leq \varepsilon|t|+C_{\varepsilon}|t|^{p-1}
$$

and

$$
|F(x, t)|,|G(x, t)| \leq \varepsilon t^{2}+C_{\varepsilon}|t|^{p}
$$

for all $(x, t)$, where $p>2$. Then for $z^{+}=(u, u)$ with $\left\|z^{+}\right\|$small

$$
\begin{aligned}
\Phi(z) & =\Phi(u, u)=\frac{1}{2}\left\|z^{+}\right\|^{2}-\Psi\left(z^{+}\right) \\
& =\frac{1}{2}\left\|z^{+}\right\|^{2}-\int_{\mathbb{R}^{N}} F(x, u)+G(x, u) \\
& =\left(\frac{1}{2}-\varepsilon\right)\left\|z^{+}\right\|^{2}-o\left(\left\|z^{+}\right\|^{2}\right) .
\end{aligned}
$$

Now, the conclusion follows.

We claim that $\mu:=\inf \sigma(S) \leq \max V$. If not, we can choose $u_{n} \in \mathcal{D}(S)$ with $\left|u_{n}\right|_{2}=1$ and $\left|-\Delta u_{n}\right|_{2} \rightarrow 0$ since $\sigma(-\Delta)=[0, \infty)$. Then $\left|\nabla u_{n}\right|_{2} \rightarrow 0$ and

$$
\begin{aligned}
\max V & <\underline{\mu}=\underline{\mu}\left|u_{n}\right|_{2} \leq\left(S u_{n}, u_{n}\right)_{L^{2}}=\left((-\Delta+V(x)) u_{n}, u_{n}\right)_{L^{2}} \\
& \leq\left|\nabla u_{n}\right|_{2}^{2}+\max V \rightarrow \max V,
\end{aligned}
$$

which is a contradiction. By $\left(\mathrm{H}_{3}\right)$, we can take a number $\gamma$ such that

$$
\underline{\mu} \leq \max V<\gamma<\min \{l, m\} .
$$

Since $\sigma(S)$ is absolutely continuous, the subspace $Y_{0}:=\left(E_{\gamma}-E_{0}\right) L^{2}$ is infinite dimensional, where $\left\{E_{\lambda}\right\}_{\lambda \in \mathbb{R}}$ is the spectral family of $S$. Then

$$
\underline{\mu}|u|_{2}^{2} \leq\|u\|_{V}^{2} \leq \gamma|u|_{2}^{2} \text { for all } u \in Y_{0} .
$$

Letting $W_{0}:=\left\{(u, u) \mid u \in Y_{0}\right\}$, then

$$
\underline{\mu}|w|_{2}^{2} \leq\|w\|^{2} \leq \gamma|w|_{2}^{2} \quad \text { for all } \quad w \in W_{0} .
$$

Let $\left\{\gamma_{n}\right\}$ be a sequence with

$$
\underline{\mu}=\gamma_{0}<\gamma_{1}<\gamma_{2}<\cdots \leq \gamma .
$$

For each $n \in \mathbb{N}$, take an element $e_{n} \in\left(E_{\gamma_{n}}-E_{\gamma_{n-1}}\right) L^{2}$ with $\left\|e_{n}\right\|=1$ and define $Y_{n}:=\operatorname{span}\left\{e_{1}, \ldots, e_{n}\right\}$. Then $W_{n}:=\left\{(u, u): u \in Y_{n}\right\} \subset W_{0}$ is an increasing sequence of finite dimensional subspace of $E^{+}$. Set $E_{n}=E^{-} \oplus W_{n}$. 
Lemma 3.2. Let $\left(\mathrm{H}_{0}\right)$ and $\left(\mathrm{H}_{2}\right)-\left(\mathrm{H}_{3}\right)$ be satisfied and $\rho>0$ be given by Lemma 3.1. Then $\sup \Phi\left(E_{n}\right)<\infty$, and there is a sequence $R_{n}>0$ such that $\sup \Phi\left(E_{n} \backslash B_{n}\right)<$ $\inf \Phi\left(B_{\rho} \cap E^{+}\right)$, where $B_{n}:=\left\{z \in E_{n}:\|z\| \leq R_{n}\right\}$.

Proof. It is sufficient to prove that $\Phi(z) \rightarrow-\infty$ in $E_{n}$ as $\|z\| \rightarrow \infty$. If not, then there are $M>0$ and $\left\{z_{j}\right\} \subset E_{n}$ with $\left\|z_{j}\right\| \rightarrow \infty$ such that $\Phi\left(z_{j}\right) \geq-M$ for all $j$, where $z_{j}=\left(u_{j}, v_{j}\right)$. Denote $y_{j}:=z_{j} /\left\|z_{j}\right\|=\left(u_{j} /\left\|z_{j}\right\|, v_{j} /\left\|z_{j}\right\|\right)$, passing to a subsequence if necessary, $y_{j} \rightarrow y=(\varphi, \psi), y_{j}^{-} \rightarrow y^{-}$and $y_{j}^{+} \rightarrow y^{+}$. Now we have

$$
\begin{aligned}
\frac{1}{2}\left(\left\|y_{j}^{+}\right\|^{2}-\left\|y_{j}^{-}\right\|^{2}\right) & \geq \frac{1}{2}\left(\left\|y_{j}^{+}\right\|^{2}-\left\|y_{j}^{-}\right\|^{2}\right)-\frac{\Psi\left(z_{j}\right)}{\left\|z_{j}\right\|^{2}} \\
& =\frac{\Phi\left(z_{j}\right)}{\left\|z_{j}\right\|^{2}} \geq \frac{-M}{\left\|z_{j}\right\|^{2}},
\end{aligned}
$$

here we use the fact that, since $F, G \geq 0, \Psi(z) \geq 0$, which yields that

$$
\frac{1}{2}\left\|y_{j}^{-}\right\|^{2} \leq \frac{1}{2}\left\|y_{j}^{+}\right\|^{2}+\frac{M}{\left\|z_{j}\right\|^{2}} .
$$

We claim that $y^{+} \neq 0$. Indeed, if not it follows from (3.6) that $\left\|y_{j}^{-}\right\| \rightarrow 0$. Thus $\left\|y_{j}\right\| \rightarrow 0$, which contradicts with $\left\|y_{j}\right\|=1$. By (3.3) we get

$$
\begin{aligned}
\left\|y^{+}\right\|^{2}-\left\|y^{-}\right\|^{2}-\int_{\mathbb{R}^{N}} l|\varphi|^{2}+m|\psi|^{2} & \leq\left\|y^{+}\right\|^{2}-\left\|y^{-}\right\|^{2}-\min \{l, m\}|y|_{2}^{2} \\
& \leq-(\min \{l, m\}-\gamma)\left|y^{+}\right|_{2}^{2}<0,
\end{aligned}
$$

from where it follows that there exist $\Omega_{1}, \Omega_{2} \subset \mathbb{R}^{N}$ such that

$$
\left\|y^{+}\right\|^{2}-\left\|y^{-}\right\|^{2}-l \int_{\Omega_{1}}|\varphi|^{2}-m \int_{\Omega_{2}}|\psi|^{2}<0 .
$$

Setting $r_{1}(x, t):=f(x, t)-l t, r_{2}(x, t):=g(x, t)-m t$ and $R_{i}(x, t)=\int_{0}^{t} r_{i}(x, s) d s$ for $i=1,2$, then $\left|r_{i}(x, t)\right| \leq c|t|$ for some $c>0, r_{i}(x, t) /|t| \rightarrow 0$ as $|t| \rightarrow \infty$ uniformly in $x$, where $i=1,2$. Since $\left\|z_{j}\right\| \rightarrow \infty,\left\|u_{j}\right\|_{V}^{2}+\left\|v_{j}\right\|_{V}^{2} \rightarrow \infty$, and hence at least one of $\left\|u_{j}\right\|_{V}$ and $\left\|v_{j}\right\|_{V}$ will convergent to infinity as $j \rightarrow \infty$. Without loss of generality, we assume that $\left\|u_{j}\right\|_{V} \rightarrow \infty$ and $\left\|v_{j}\right\|_{V}$ is bounded. The other two cases are similar. Note that

$$
\frac{\left|R_{1}\left(x, u_{j}\right)\right|}{\left\|z_{j}\right\|^{2}} \leq \frac{\left|R_{1}\left(x, u_{j}\right)\right|}{\left\|u_{j}\right\|_{V}^{2}}=\frac{\left|R_{1}\left(x, u_{j}\right)\right|}{\left|u_{j}\right|^{2}} \frac{\left|u_{j}\right|^{2}}{\left\|u_{j}\right\|_{V}^{2}},
$$

and $\frac{\left|R_{1}\left(x, u_{j}\right)\right|}{\left|u_{j}\right|^{2}} \frac{\left|u_{j}\right|^{2}}{\left\|u_{j}\right\|_{V}^{2}} \rightarrow 0$ a.e. on $\mathbb{R}^{N}$ and $\frac{\left|R_{1}\left(x, u_{j}\right)\right|}{\left|u_{j}\right|^{2}} \frac{\left|u_{j}\right|^{2}}{\left\|u_{j}\right\|_{V}^{2}} \leq C \frac{\left|u_{j}\right|^{2}}{\left\|u_{j}\right\|_{V}^{2}}$. From dominate convergence theorem we obtain that

$$
\lim _{j \rightarrow \infty} \int_{\Omega_{1}} \frac{\left|R_{1}\left(x, u_{j}\right)\right|}{\left\|z_{j}\right\|^{2}}=0 .
$$


Similarly, using dominate convergence theorem again and the boundedness of $R_{2}\left(x, v_{j}\right)$ one has

$$
\lim _{j \rightarrow \infty} \int_{\Omega_{2}} \frac{\left|R_{2}\left(x, v_{j}\right)\right|}{\left\|z_{j}\right\|^{2}} \leq \lim _{j \rightarrow \infty} \int_{\Omega_{2}} \frac{C v_{j}^{2}}{\left\|z_{j}\right\|^{2}} \leq \lim _{j \rightarrow \infty} \frac{M}{\left\|z_{j}\right\|^{2}}=0 .
$$

Thus (3.5), (3.7)-(3.9) imply that

$$
\begin{aligned}
0 & \leq \lim _{j \rightarrow \infty}\left[\frac{1}{2}\left(\left\|y_{j}^{+}\right\|^{2}-\left\|y_{j}^{-}\right\|^{2}\right)-\int_{\Omega_{1}} \frac{F\left(x, u_{j}\right)}{\left\|z_{j}\right\|^{2}}-\int_{\Omega_{1}} \frac{G\left(x, v_{j}\right)}{\left\|z_{j}\right\|^{2}}\right] \\
& =\lim _{j \rightarrow \infty}\left[\frac{1}{2}\left(\left\|y_{j}^{+}\right\|^{2}-\left\|y_{j}^{-}\right\|^{2}\right)-\int_{\Omega_{1}} \frac{\frac{1}{2} l u_{j}^{2}+R_{1}\left(x, u_{j}\right)}{\left\|z_{j}\right\|^{2}}-\int_{\Omega_{1}} \frac{\frac{1}{2} m v_{j}^{2}+R_{2}\left(x, v_{j}\right)}{\left\|z_{j}\right\|^{2}}\right] \\
& \leq \frac{1}{2}\left(\left\|y^{+}\right\|^{2}-\left\|y^{-}\right\|^{2}-l \int_{\Omega_{1}}|\varphi|^{2}-m \int_{\Omega_{2}}|\psi|^{2}\right)<0 .
\end{aligned}
$$

Now the desired conclusion follows from this contradiction.

As a consequence, we have:

Lemma 3.3. Let $\left(H_{0}\right)$ and $\left(H_{2}\right)-\left(H_{3}\right)$ be satisfied and $\kappa>0$ be given by Lemma 3.1 . Then letting $e \in W_{0}$ with $\|e\|=1$, there is $R_{1}>0$ such that $\left.\Phi\right|_{\partial Q} \leq \kappa$, where $Q:=\left\{z=z^{-}+s e: z^{-} \in E^{-}, s \geq 0,\|z\| \leq R_{1}\right\}$

\section{The $(C)_{c}$-sequence}

Lemma 4.1. Suppose that $\left(H_{0}\right)-\left(H_{2}\right)$ and $\left(H_{4}\right)-\left(H_{5}\right)$ are satisfied. Then any $(C)_{c^{-}}$ sequence of $\Phi$ is bounded.

Proof. Let $\left\{z_{j}\right\}$ be such that $\Phi\left(z_{j}\right) \rightarrow c$ and $\left(1+\left\|z_{j}\right\|\right) \Phi^{\prime}\left(z_{j}\right) \rightarrow 0$, write $z_{j}=$ $\left(u_{j}, v_{j}\right)$. Suppose to the contrary that $\left\{z_{j}\right\}$ is unbounded. Setting $y_{j}:=z_{j} /\left\|z_{j}\right\|$, then $\left\|y_{j}\right\|=1$. In what follows, we write $y_{j}=\left(u_{j} /\left\|z_{j}\right\|, v_{j} /\left\|z_{j}\right\|\right):=\left(\varphi_{j}, \psi_{j}\right)$. Without lose of generality, we can assume that $y_{j} \rightarrow y=(\varphi, \psi)$ in $E$. Observe that for $j$ large

$$
C \geq \Phi\left(z_{j}\right)-\frac{1}{2} \Phi^{\prime}\left(z_{j}\right) z_{j}=\int_{\mathbb{R}^{N}} \hat{F}\left(x, u_{j}\right)+\hat{G}\left(x, v_{j}\right),
$$

and

$$
\begin{aligned}
\Phi^{\prime}\left(z_{j}\right)\left(z_{j}^{+}-z_{j}^{-}\right) & =\left\|z_{j}\right\|^{2}-\Psi^{\prime}\left(z_{j}\right)\left(z_{j}^{+}-z_{j}^{-}\right) \\
& =\left\|z_{j}\right\|^{2}-\Psi^{\prime}\left(u_{j}, v_{j}\right)\left(v_{j}, u_{j}\right) \\
& =\left\|z_{j}\right\|^{2}\left[1-\int_{\mathbb{R}^{N}} \frac{v_{j} f\left(x, u_{j}\right)+u_{j} g\left(x, v_{j}\right)}{\left\|z_{j}\right\|^{2}}\right] .
\end{aligned}
$$

From (4.2),

$$
\int_{\mathbb{R}^{N}} \frac{v_{j} f\left(x, u_{j}\right)+u_{j} g\left(x, v_{j}\right)}{\left\|z_{j}\right\|^{2}} \rightarrow 1
$$


By $\left(H_{0}\right)-\left(H_{2}\right)$, there is some $C>0$ such that

$$
|f(x, t)| \leq C|t| \quad \text { and } \quad|g(x, t)| \leq C|t|
$$

for all $(x, t)$. Without restriction we can assume that $\left\{y_{j}\right\}$ is either vanishing or nonvanishing. We shall prove that both cases are impossible and this will give us the desired contradiction.

If $\left\{y_{j}\right\}$ is vanishing, then by the vanishing lemma we have $\varphi_{j} \rightarrow 0$ and $\psi_{j} \rightarrow 0$ in $\left.L^{p}\left(\mathbb{R}^{N}\right)\right)$ for $p \in\left(2,2^{*}\right)$. In virtue of $\left(H_{5}\right)$, set

$$
\Omega_{j}:=\left\{x \in \mathbb{R}^{N} \mid \frac{\left|f\left(x, u_{j}(x)\right)\right|}{\left|u_{j}(x)\right|} \leq V_{0}-\delta_{0} \text { and } \frac{\left|g\left(x, v_{j}(x)\right)\right|}{\left|v_{j}(x)\right|} \leq V_{0}-\delta_{0}\right\} .
$$

Observe that $V_{0}\left|\varphi_{j}\right|_{2}^{2} \leq\left\|\varphi_{j}\right\|_{V}^{2}$ and $V_{0}\left|\psi_{j}\right|_{2}^{2} \leq\left\|\psi_{j}\right\|_{V}^{2}$ since $\sigma(S) \subset\left[V_{0}, \infty\right)$. Hence $V_{0}\left|y_{j}\right|_{2}^{2} \leq\left\|y_{j}\right\|^{2}=1$, from where we have

$$
\begin{aligned}
\left|\int_{\Omega_{j}} \frac{v_{j} f\left(x, u_{j}\right)+u_{j} g\left(x, v_{j}\right)}{\left\|z_{j}\right\|^{2}}\right| & \leq 2\left(V_{0}-\delta_{0}\right) \int_{\Omega_{j}}\left|\varphi_{j} \psi_{j}\right| \\
& \leq\left(V_{0}-\delta_{0}\right)\left|y_{j}\right|_{2}^{2} \\
& \leq \frac{V_{0}-\delta_{0}}{V_{0}}
\end{aligned}
$$

for all $j$. This, jointly with (4.3), implies that for $\Omega_{j}^{c}=\mathbb{R}^{N} \backslash \Omega_{j}$

$$
\lim _{j \rightarrow \infty} \int_{\Omega_{j}^{c}} \frac{v_{j} f\left(x, u_{j}\right)+u_{j} g\left(x, v_{j}\right)}{\left\|z_{j}\right\|^{2}}>1-\frac{V_{0}-\delta_{0}}{V_{0}}=\frac{\delta_{0}}{V_{0}} .
$$

Then by (4.4) there holds for an arbitrarily fixed $s \in\left(2,2^{*}\right)$,

$$
\begin{aligned}
\int_{\Omega_{j}^{c}} \frac{v_{j} f\left(x, u_{j}\right)+u_{j} g\left(x, v_{j}\right)}{\left\|z_{j}\right\|^{2}} & \leq 2 \int_{\Omega_{j}^{c}}\left|\varphi_{j} \psi_{j}\right| \\
& \leq C\left|\varphi_{j}\right|_{2}\left|\Omega_{j}^{c}\right|^{(s-2) / 2 s}\left|\psi_{j}\right|_{s} .
\end{aligned}
$$

Since $\left|\psi_{j}\right|_{s} \rightarrow 0$ and $\left|\varphi_{j}\right|_{2}$ is bounded, one has $\left|\Omega_{j}^{c}\right| \rightarrow \infty$. From $\left(H_{5}\right)$, at least one of $\hat{F}(x, t) \geq \delta_{0}$ and $\hat{G}(x, t) \geq \delta_{0}$ hold on $\Omega_{j}^{c}$ and hence

$$
\int_{\mathbb{R}^{N}} \hat{F}\left(x, u_{j}\right)+\hat{G}\left(x, v_{j}\right) \geq \int_{\Omega_{j}^{c}} \hat{F}\left(x, u_{j}\right)+\hat{G}\left(x, v_{j}\right) \geq \delta_{0}\left|\Omega_{j}^{c}\right| \rightarrow \infty,
$$

contrary to $(4.1)$.

If $\left\{y_{j}\right\}$ is nonvanishing, i.e., there exist $\alpha>0, R<\infty$ and $\left\{a_{j}\right\} \subset \mathbb{R}^{N}$ such that

$$
\liminf _{j \rightarrow \infty} \int_{B\left(a_{j}, R\right)}\left|y_{j}\right|^{2} \geq \alpha .
$$

Setting $\tilde{z}_{j}(x)=z_{j}\left(x+a_{j}\right)=\left(u_{j}\left(x+a_{j}\right), v_{j}\left(x+a_{j}\right)\right):=\left(\tilde{u}_{j}(x), \tilde{v}_{j}(x)\right), \tilde{y}_{j}(x)=$ $y_{j}\left(x+a_{j}\right)=\left(\tilde{u}_{j}(x) /\left\|z_{j}\right\|, \tilde{v}_{j}(x) /\left\|z_{j}\right\|\right), \eta=(\varphi, \psi), \eta_{j}(x)=\left(\varphi_{j}(x), \psi_{j}(x)\right):=$ 
$\left(\left(\varphi\left(x+a_{j}\right), \psi\left(x+a_{j}\right)\right)\right.$ for any $\varphi, \psi \in C_{0}^{\infty}\left(\mathbb{R}^{N}\right)$, from $\left(H_{2}\right)$ we have

$$
\begin{aligned}
o(1) & =\Phi^{\prime}\left(z_{j}\right) \eta_{j} \\
& =\left(z_{j}^{+}-z_{j}^{-}, \eta_{j}\right)-\int_{\mathbb{R}^{N}} f\left(x, u_{j}\right) \varphi_{j}+g\left(x, v_{j}\right) \psi_{j} \\
& =\left\|z_{j}\right\|\left[\left(\tilde{y}_{j}^{+}-\tilde{y}_{j}^{-}, \eta\right)-\int_{\mathbb{R}^{N}} \frac{l \tilde{u}_{j} \varphi+m \tilde{v}_{j} \psi}{\left\|z_{j}\right\|}-\int_{\mathbb{R}^{N}} \frac{r_{1}\left(x, \tilde{u}_{j}\right) \varphi+r_{2}\left(x, \tilde{v}_{j}\right) \psi}{\left\|z_{j}\right\|}\right] .
\end{aligned}
$$

Since $\left\|\tilde{y}_{j}\right\|=\left\|y_{j}\right\|=1$, up to a subsequence we assume that $\tilde{y}_{j} \rightarrow \tilde{y}=(\zeta, \xi)$ in $E$, $\tilde{y}_{j} \rightarrow \tilde{y}$ in $L_{\text {loc }}^{2}\left(\mathbb{R}^{N}, \mathbb{R}\right) \times L_{\text {loc }}^{2}\left(\mathbb{R}^{N}, \mathbb{R}\right)$ and $\tilde{y}_{j}(x) \rightarrow \tilde{y}(x)$ a.e. in $\mathbb{R}^{N}$. Clearly, $\tilde{y} \neq 0$. Similar to (3.8) and (3.9), one has

$$
\int_{\mathbb{R}^{N}} \frac{r_{1}\left(x, \tilde{u}_{j}\right) \varphi+r_{2}\left(x, \tilde{v}_{j}\right) \psi}{\left\|z_{j}\right\|}=o(1) .
$$

Letting $j \rightarrow \infty$ in (4.5) we obtain that

$$
\left(\tilde{y}^{+}-\tilde{y}^{-}, \eta\right)-l(\zeta, \varphi)_{L^{2}}-m(\xi, \psi)_{L^{2}}=0,
$$

which is equivalent to

$$
\int_{\mathbb{R}^{N}} \nabla \xi \nabla \varphi+V(x) \xi \varphi+\nabla \zeta \nabla \psi+V(x) \zeta \psi-\int_{\mathbb{R}^{N}} l \zeta \varphi+m \xi \psi=0,
$$

from where it follows that $(\zeta, \xi)$ is critical point of the functional

$$
I(u, v)=\int_{\mathbb{R}^{N}} \nabla u \nabla v+V(x) u v-\int_{\mathbb{R}^{N}} l u+m v,
$$

i.e., $(\zeta, \xi)$ is a weak solution of the following system

$$
\left\{\begin{array}{l}
-\Delta u+V(x) u=m v \text { in } \mathbb{R}^{N}, \\
-\Delta v+V(x) v=l u \text { in } \mathbb{R}^{N} .
\end{array}\right.
$$

As in Proposition 2.4 of $\mathrm{Li}$ and Yang [21], we know that $(\zeta, \sqrt{m / l} \xi)$ is a weak solution of

$$
\left\{\begin{aligned}
-\Delta u+V(x) u & =\sqrt{\operatorname{lm}} v, \\
-\Delta v+V(x) v & =\sqrt{\operatorname{lm} u} .
\end{aligned}\right.
$$

If $\zeta \neq \sqrt{m / l} \xi$, then $-\Delta(\zeta-\sqrt{m / l} \xi)+V(x)(\zeta-\sqrt{m / l} \xi)=-\sqrt{l m}(\zeta-\sqrt{m / l} \xi)$. Hence $-\sqrt{l m}$ is an eigenvalue of $S$, contradicts with $S$ has only continuous spectrum. If $\zeta=\sqrt{m / l} \xi \neq 0$, then $-\Delta \zeta+V(x) \zeta=\sqrt{l m} \zeta$. Thus $\sqrt{l m}$ is also a eigenvalue of $S$, which is impossible, whence $\zeta=\xi=0$, on the contrary to the fact that $\tilde{y} \neq 0$. Therefore $\left\{z_{j}\right\}$ is bounded in $E$.

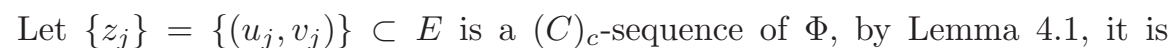
bounded, up to a subsequence, we may assume $u_{j} \rightarrow u$ and $v_{j} \rightarrow v$ in $H^{1}\left(\mathbb{R}^{N}\right)$, $u_{j} \rightarrow u$ and $v_{j} \rightarrow v$ in $L_{l o c}^{p}\left(\mathbb{R}^{N}\right)$ for $p \in\left[2,2^{*}\right)$ and $z_{j}(x) \rightarrow z(x)=(u(x), v(x))$ a.e. on $\mathbb{R}^{N}$. Plainly, $z$ is a critical point of $\Phi$.

Recall a mapping $f$ from Banach space $X$ to another Banach space $Y$ is called BL-splits, if for every sequence $\left\{x_{n}\right\}$ in $X$ with $x_{n} \rightarrow x$ it holds that $f\left(x_{n}\right)-f\left(x_{n}-\right.$ $x) \rightarrow f(x)$ in $Y$ (see Ackermann [2]). We adopt here a cut-off technique developed 
in Ackermann [1]. Let $\eta:[0, \infty) \rightarrow[0,1]$ be a smooth function satisfying $\eta(s)=1$ if $s \leq 1, \eta(s)=0$ if $s \geq 2$. Define $\tilde{z}_{j}(x)=\eta(2|x| / j) z(x)$, then $\tilde{z}_{j} \rightarrow z$ in $E$. In a similar way to Lemma 3.2 in Ackermann [1] (see also Lemma 4.4 in Ding and Jeanjean [15]), we can obtain the following

Lemma 4.2. Under the assumptions of Theorem 1.1, $\Psi(\cdot)$ and $\Psi^{\prime}(\cdot)$ are both BL-splits.

Let $\mathcal{K}:=\left\{z \in E \mid \Phi^{\prime}(z)=0, z \neq 0\right\}$ be the set of nontrivial critical points of $\Phi$. According to the first step in the proof of Theorem 1.1 in the next section, $\mathcal{K}$ is not empty.

Lemma 4.3. Under the assumptions of Lemma 4.1, the following two conclusions hold

$$
\begin{aligned}
& \text { (1) } \quad \nu:=\inf \{\|z\|: z \in \mathcal{K}\}>0 ; \\
& \text { (2) } \quad \theta:=\inf \{\Phi(z) \mid z \in \mathcal{K}\}>0 .
\end{aligned}
$$

Proof. (1) For any $z=(u, v) \in \mathcal{K}$, there holds

$$
0=\Phi^{\prime}(z)\left(z^{+}-z^{-}\right)=\|z\|^{2}-\int_{\mathbb{R}^{N}} v f(x, u)+u g(x, v),
$$

jointly with (3.1), which implies that

$$
\|z\|^{2} \leq \varepsilon|z|_{2}^{2}+C_{\varepsilon}|z|_{p}^{p} \leq c \varepsilon\|z\|_{2}^{2}+c C_{\varepsilon}\|z\|^{p},
$$

where $p \in\left(2,2^{*}\right)$. Choose $\varepsilon$ small enough, hence

$$
0<\left(\frac{1-c \varepsilon}{c C_{\varepsilon}}\right)^{\frac{1}{p-2}} \leq\|z\|
$$

for each $z \in \mathcal{K}$.

(2) Suppose to the contrary that there exist a sequence $\left\{z_{j}\right\}=\left\{\left(u_{j}, v_{j}\right)\right\} \in \mathcal{K}$ such that $\Phi\left(z_{j}\right) \rightarrow 0$. By (1), $\left\|z_{j}\right\| \geq \nu$. Clearly, $\left\{z_{j}\right\}$ is a $(C)_{0}$-sequence of $\Phi$, and hence is bounded from Lemma 4.1. Moreover, $\left\{z_{j}\right\}$ is nonvanishing. By the invariance under translation of $\Phi$, we can assume, up to a translation, that $z_{j} \rightarrow$ $z=(u, v) \in \mathcal{K}$. Since $z=(u, v)$ is a solution of (ES), $|u(x)| \rightarrow 0$ and $|v(x)| \rightarrow 0$ as $|x| \rightarrow \infty$. Since $\|z\|^{2}=\|u\|_{V}^{2}+\|v\|_{V}^{2} \geq \nu$, without loss of generality, there is a bounded domain $\Omega \subset \mathbb{R}^{N}$ with positive measure such that $0<|u(x)|<\delta_{1}$ for $x \in \Omega$ by $\left(H_{5}\right)$. Then

$$
o(1)=\Phi\left(z_{j}\right)=\Phi\left(z_{j}\right)-\frac{1}{2} \Phi^{\prime}\left(z_{j}\right) z_{j}=\int_{\mathbb{R}^{N}} \hat{F}\left(x, u_{j}\right)+\hat{G}\left(x, v_{j}\right) \geq \int_{\Omega} \hat{F}\left(x, u_{j}\right),
$$

letting $j \rightarrow \infty$ yields

$$
0 \geq \lim _{j \rightarrow \infty} \int_{\Omega} \hat{F}\left(x, u_{j}\right) \geq \int_{\Omega} \hat{F}(x, u)>0 .
$$

This ends the proof. 
In the following lemma we discuss further the $(C)_{c}$-sequence. Let $[l]$ denote the integer part of $l \in \mathbb{R}$. The following lemma is standard (see Coti Zelati and Rabinowitz [9,10] and Séré [24]).

Lemma 4.4. Under the assumptions of Theorem 1.1, let $\left\{z_{j}\right\} \subset E$ be a $(C)_{c^{-}}$ sequence of $\Phi$. Then either

(i) $z_{j} \rightarrow 0$ (and hence $c=0$ ), or

(ii) $c \geq \theta$ and there exist a positive integer $l \leq[c / \theta], y_{1}, \ldots, y_{l} \in \mathcal{K}$ and sequences $\left\{a_{j}^{i}\right\} \subset \mathbb{Z}^{N}, i=1,2, \ldots, l$, such that, after extraction of a subsequence of $\left\{z_{j}\right\}$,

$$
\begin{aligned}
\left\|z_{j}-\sum_{i=1}^{l} a_{j}^{i} * y_{i}\right\| & \rightarrow 0, \\
\sum_{i=1}^{k} \Phi\left(y_{i}\right) & =c
\end{aligned}
$$

and for $i \neq k$,

$$
\left|a_{j}^{i}-a_{j}^{l}\right| \rightarrow \infty
$$

as $j \rightarrow \infty$.

\section{Proof of Theorem 1.1}

In this section we prove Theorem 1.1. First, we recall some notations. Let $E$ be a Banach space with direct sum $E=X \oplus Y$ and corresponding projections $P_{X}, P_{Y}$ onto $X, Y$. Let $\mathcal{S} \subset X^{*}$ be a dense subset, for each $s \in \mathcal{S}$ there is a semi-norm on $E$ defined by

$$
p_{s}: E \rightarrow \mathbb{R}, p_{s}(u)=|s(x)|+\|y\| \quad \text { for } \quad u=x+y \in E .
$$

We denote by $\mathcal{T}_{\mathcal{S}}$ the topology induced by semi-norm family $\left\{p_{s}\right\}, w^{*}$ denote the weak*-topology on $E^{*}$. For a functional $\Phi \in C^{1}(E, \mathbb{R})$ we write $\Phi_{a}=\{u \in$ $E \mid \Phi(u) \geq a\}, \Phi^{b}=\{u \in E \mid \Phi(u) \leq b\}$ and $\Phi_{a}^{b}=\Phi_{a} \cap \Phi^{b}$. A set $\mathcal{A} \subset E$ is said to be a $(C)_{c}$-attractor if for any $\varepsilon, \delta>0$ and any $(C)_{c}$-sequence $\left\{z_{j}\right\}$ there is $j_{0}$ such that $z_{j} \in U_{\varepsilon}\left(\mathcal{A} \cap \Phi_{c-\delta}^{c+\delta}\right)$ for $j \geq j_{0}$. Given an interval $I \subset \mathbb{R}, \mathcal{A}$ is said to be a $(C)_{I^{-}}$ attractor if it is a $(C)_{c}$-attractor for all $c \in I$. $\Phi$ is said to be weakly sequentially lower semicontinuous if for any $u_{j} \rightarrow u$ in $E$ one has $\Phi(u) \leq \liminf _{j \rightarrow \infty} \Phi\left(u_{j}\right)$, and $\Phi^{\prime}$ is said to be weakly sequentially continuous if $\lim _{j \rightarrow \infty} \Phi^{\prime}\left(u_{j}\right) w=\Phi^{\prime}(u) w$ for each $w \in E$. Suppose

$\left(\Phi_{0}\right)$ for any $c \in \mathbb{R}, \Phi_{c}$ is $\mathcal{T}_{\mathcal{S}}$-closed, and $\Phi^{\prime}:\left(\Phi_{c}, \mathcal{T}_{\mathcal{S}}\right) \rightarrow\left(E^{*}, w^{*}\right)$ is continuous;

$\left(\Phi_{1}\right)$ for any $c>0$, there exists $\xi>0$ such that $\|u\|<\xi\left\|P_{Y} u\right\|$ for all $u \in \Phi_{c}$;

$\left(\Phi_{2}\right)$ there exists $\rho>0$ such that $\kappa:=\inf \Phi\left(S_{\rho} \cap Y\right)>0$, where $S_{\rho}:=\{u \in E$ : $\|u\|=\rho\}$

$\left(\Phi_{3}\right)$ There is an increasing sequence $Y_{n} \subset Y$ of finite-dimensional subspaces and a sequence $\left\{R_{n}\right\}$ of positive numbers such that, letting $E_{n}=X \oplus Y_{n}$ and $B_{n}=B_{R_{n}} \cap E_{n}, \sup \Phi\left(E_{n}\right)<\infty$ and $\sup \Phi\left(E_{n} \backslash B_{n}\right)<\inf \Phi\left(B_{\rho} \cap Y\right) ;$ 
$\left(\Phi_{4}\right)$ For any interval $I \subset(0, \infty)$ there is a $(C)_{I}$-attractor $A$ with $P_{X} A$ bounded and $\inf \left\{\left\|P_{Y}(z-w): z, w \in A, P_{Y}(z-w) \neq 0\right\|\right\}>0$.

Now we state two critical point theorems which will be used later (see BartschDing [7]).

Theorem 5.1. Let $\left(\Phi_{0}\right)-\left(\Phi_{2}\right)$ be satisfied and suppose there are $R>\rho>0$ and $e \in Y$ with $\|e\|=1$ such that $\sup \Phi(\partial Q) \leq \kappa$ where $Q:=\{u=x+$ te $: x \in X, t \geq$ $0,\|u\|<R\}$. Then $\Phi$ has a $(C)_{c}$-sequence with $\kappa \leq c \leq \sup \Phi(Q)$.

Theorem 5.2. Assume $\Phi$ is even with $\Phi(0)=0$, let $\left(\Phi_{0}\right)-\left(\Phi_{4}\right)$ be satisfied. Then $\Phi$ has possesses an unbounded sequence of positive critical values.

Lemma 5.3. $\Phi$ defined in (2.1) satisfies $\left(\Phi_{0}\right)$.

Proof. We first show that $\Phi_{a}$ is $\mathcal{T}_{S}$-closed for every $a \in \mathbb{R}$. Consider a sequence $\left\{z_{j}\right\}=\left\{\left(u_{j}, v_{j}\right)\right\} \subset \Phi_{a}$ which $\mathcal{T}_{S^{-}}$-converges to $z=(u, v) \in E$, and write $z_{j}=$ $z_{j}^{-}+z_{j}^{+}, z=z^{-}+z^{+}$. Then $z_{j}^{+} \rightarrow z^{+}$in norm topology and hence $\left\{z_{j}^{+}\right\}$is bounded in norm topology. Observe that there exists $C>0$ such that

$$
\left\|z_{j}^{-}\right\|^{2}=\left\|z_{j}^{+}\right\|^{2}-2 \Phi\left(z_{j}\right)-2 \int_{\mathbb{R}^{N}} F\left(x, u_{j}\right)+G\left(x, v_{j}\right) \leq C
$$

since $F(x, t), G(x, t) \geq 0$. This implies the boundedness of $\left\{z_{j}^{-}\right\}$and hence $z_{j}^{-} \rightarrow$ $z^{-}$. Therefore we have $u_{j} \rightarrow u$ and $v_{j} \rightarrow v$. It is easy to check that $\Psi$ is weakly sequentially lower semi-continuous by the fact that $H^{1}\left(\mathbb{R}^{N}\right) \hookrightarrow L_{l o c}^{p}\left(\mathbb{R}^{N}\right)$ for $p \in$ $\left[2,2^{*}\right)$ and the Fatou's Lemma, from which it follows that

$$
a \leq \lim _{n \rightarrow \infty} \Phi\left(z_{j}\right) \leq \Phi(z),
$$

so $z \in \Phi_{a}$ and hence $\Phi_{a}$ is $\mathcal{T}_{S}$-closed.

Next we show that $\Phi^{\prime}:\left(\Phi_{c}, \mathcal{T}_{\mathcal{S}}\right) \rightarrow\left(E^{*}, w^{*}\right)$ is continuous. It is suffices to show that $\Psi^{\prime}$ has the same property. Suppose $z_{j}=\left(u_{j}, v_{j}\right) \rightarrow z=(u, v)$ in $\mathcal{T}_{\mathcal{S}}$ topology. Then $u_{j} \rightarrow u$ and $v_{j} \rightarrow v$ in $L_{l o c}^{p}\left(\mathbb{R}^{N}\right)$ for $p \in\left[2,2^{*}\right)$. It is obvious that

$\Psi^{\prime}\left(z_{j}\right)(\varphi, \psi)=\int_{\mathbb{R}^{N}} f\left(x, u_{j}\right) \varphi+g\left(x, v_{j}\right) \psi \rightarrow \int_{\mathbb{R}^{N}} f(x, u) \varphi+g(x, v) \psi=\Psi^{\prime}(z)(\varphi, \psi)$, as $n \rightarrow \infty$ for each $\varphi, \psi \in C_{0}^{\infty}\left(\mathbb{R}^{N}\right)$. Now using the density of $C_{0}^{\infty}\left(\mathbb{R}^{N}\right)$ in $H^{1}\left(\mathbb{R}^{N}\right)$ we can obtain the desired conclusion.

Lemma 5.4. $\Phi$ satisfies $\left(\Phi_{1}\right)$.

Proof. For any $c>0$ and $z \in \Phi_{c}$, using the fact that $\Psi \geq 0$ one has

$$
0<c \leq \frac{1}{2}\left(\left\|z^{+}\right\|^{2}-\left\|z^{-}\right\|^{2}\right) .
$$

This yields $\left\|z^{-}\right\|<\left\|z^{+}\right\|$, and hence $\|z\|^{2} \leq \sqrt{2}\left\|z^{+}\right\|$. 
Proof of Theorem 1.1. 1. Existence of positive solution. In this and next part, to obtain a positive solution, we define $f(x, t)=0$ and $g(x, t)=0$ for all $(x, t)$ with $t \leq 0$. With $X=E^{-}$and $Y=E^{+}$the condition $\left(\Phi_{0}\right)$ holds by Lemma 5.3 and $\left(\Phi_{1}\right)$ holds by Lemma 5.4. Lemma 3.1 implies $\left(\Phi_{2}\right)$. Lemma 3.3 shows that $\Phi$ possesses the linking structure of Theorem 5.1. Therefore, using Theorem 5.1, there exists a sequence $\left\{z_{j}\right\} \subset E$ such that $\Phi\left(z_{j}\right) \rightarrow c \geq \kappa$ and $\left(1+\left\|z_{j}\right\|\right) \Phi^{\prime}\left(z_{j}\right) \rightarrow 0$. By Lemma 4.1, $\left\{z_{j}\right\}$ is bounded. For the concentration functions $\left|z_{j}\right|^{2}$, there are only two cases needed to be considered: vanishing and nonvanishing. If vanishing occurs, by the vanishing lemma,

$$
\begin{aligned}
\int_{\mathbb{R}^{N}} f\left(x, u_{j}\right) u_{j} & =o(1), \quad \int_{\mathbb{R}^{N}} F\left(x, u_{j}\right)=o(1), \\
\int_{\mathbb{R}^{N}} g\left(x, v_{j}\right) v_{j} & =o(1), \quad \int_{\mathbb{R}^{N}} G\left(x, v_{j}\right)=o(1) .
\end{aligned}
$$

Then

$$
o(1)=\Phi^{\prime}\left(z_{j}\right) z_{j}=\frac{1}{2}\left(\left\|z_{j}^{+}\right\|^{2}-\left\|z_{j}^{-}\right\|^{2}\right)-\int_{\mathbb{R}^{N}}\left(f\left(x, u_{j}\right) u_{j}+g\left(x, u_{j}\right) v_{j}\right),
$$

which implies that $\frac{1}{2}\left(\left\|z_{j}^{+}\right\|^{2}-\left\|z_{j}^{-}\right\|^{2}\right)=o(1)$. Hence $\Phi\left(z_{j}\right) \rightarrow 0$ as $j \rightarrow \infty$. This contradicts to $\Phi\left(z_{j}\right) \rightarrow c \geq \kappa>0$ as $j \rightarrow \infty$. So nonvanishing occurs, i.e., there exist $\alpha>0, R<\infty$ and $\left\{a_{j}\right\} \subset \mathbb{R}^{N}$ such that

$$
\liminf _{j \rightarrow \infty} \int_{B\left(a_{j}, R\right)}\left|z_{j}\right|^{2} \geq \alpha .
$$

Setting $\tilde{z}_{j}(x)=z_{j}\left(x+a_{j}\right)$, by the invariance under translation of $\Phi,\left\{\tilde{z}_{j}\right\}$ is a $(C)_{c}$-sequence of $\Phi$ and $\tilde{z}_{j} \rightarrow \tilde{z}$. From

$$
\liminf _{j \rightarrow \infty} \int_{B(0, R)}\left|\tilde{z}_{j}\right|^{2} \geq \alpha>0,
$$

we see that $\tilde{z} \neq 0$, and hence $\tilde{z}$ is a nontrivial critical point of $\Phi$. It is obvious that $\tilde{z}=(\tilde{u}, \tilde{v})$ is a nonnegative solution of (ES) and use the strong maximum principle, $\tilde{u}$ and $\tilde{v}$ are a positive solution of (ES).

2. Existence of a least energy solution. Claim that $\theta:=\inf \{\Phi(z) \mid z \in \mathcal{K}\}$ is achieved.

In fact, the process of part 1 shows that $\mathcal{K}$ is nonempty and hence $\theta$ is finite. Let $\left\{z_{j}\right\} \subset \mathcal{K}$ is a minimizing sequence for $\theta$. Clearly, $\left\{z_{j}\right\}$ is a $(C)_{\theta}$-sequence of $\Phi$, hence is bounded by Lemma 4.1. From (1) of Lemma 4.3, $\left\|z_{j}\right\| \geq \nu>0$, one can rule out the case of vanishing. Hence $\left\{z_{j}\right\}$ is nonvanishing. Similarly, passing a translation, $\tilde{z}_{j}$ has a nonzero weak limit $\tilde{z}_{1} \in \mathcal{K}$ and $\tilde{z}_{j}(x) \rightarrow \tilde{z}_{1}(x)$ a.e. in $\mathbb{R}^{N}$, 
where $\tilde{z}_{j}$ be given as in the part 1 of proof. By Fatou's lemma,

$$
\begin{aligned}
\theta & =\lim _{j \rightarrow \infty} \Phi\left(z_{j}\right)=\lim _{j \rightarrow \infty} \Phi\left(\tilde{z}_{j}\right) \\
& \geq \liminf _{j \rightarrow \infty} \int_{\mathbb{R}^{N}} \hat{F}\left(x, \tilde{u}_{j}\right)+\hat{G}\left(x, \tilde{v}_{j}\right) \\
& \geq \int_{\mathbb{R}^{N}} \hat{F}\left(x, \tilde{u}_{1}\right)+\hat{G}\left(x, \tilde{v}_{1}\right)=\Phi\left(\tilde{z}_{1}\right),
\end{aligned}
$$

from where it follows that $\theta$ is achieved by $\tilde{z}_{1}=\left(\tilde{u}_{1}, \tilde{v}_{1}\right) \in \mathcal{K}$.

Remark 5.5. If one strengthen $\left(H_{4}\right)$ to $\left(H_{4}^{\prime}\right)$, then using the minimax character of $c$ in Remark 5.1, one can show that $c=\theta$ by a Brouwer degree argument, see Lemma 3.4 in Li and Yang [21] or Proposition 3.8 in Li and Szulkin [20] for more details. Hence $\tilde{z}$ is also a least energy solution. But in general, we do not know whether the two critical points $\tilde{z}$ and $\tilde{z}_{1}$ are same or not. $\left(H_{4}^{\prime}\right)$ guarantees that $\delta$ in $\left(H_{4}\right)$ is large enough, the latter ensures that two critical values $c$ and $\theta$ are coincide with each other.

3. Multiplicity. $\Phi$ is even provided $H(x, z)$ is even in $z$. Lemma 3.2 says that $\Phi$ satisfies $\left(\Phi_{4}\right)$. Next, we assume

$$
\mathcal{K} / \mathbb{Z}^{N} \text { is a finite set. }
$$

In fact, if (5.1) is false, then the conclusion holds automatically. In the sequel, we assume (5.1) holds. Let $\mathcal{F}$ be a set consisting of arbitrarily chosen representatives of the $\mathbb{Z}^{N}$-orbits of $\mathcal{K}$. Then $\mathcal{F}$ is a finite set by $(5.1)$, and since $\Phi^{\prime}$ is odd we may assume that $\mathcal{F}=-\mathcal{F}$. If $z \in \mathcal{K}$, then $\Phi(z) \geq \theta$ by (2) of Lemma 4.3. Hence there exists $\theta \leq \vartheta$ such that

$$
\theta \leq \min _{\mathcal{F}} \Phi=\min _{\mathcal{K}} \Phi \leq \max _{\mathcal{K}} \Phi \leq \max _{\mathcal{F}} \Phi \leq \vartheta
$$

For $l \in \mathbb{N}$ and a finite set $\mathcal{B} \subset E$ we define

$$
[\mathcal{B}, l]:=\left\{\sum_{i=1}^{j} a_{i} * z_{j} \mid 1 \leq j \leq l, a_{i} \in \mathbb{Z}^{N}, z_{i} \in \mathcal{B}\right\} .
$$

As in Coti Zelati and Rabinowitz [9] and [10],

$$
\inf \left\{\left\|z-z^{\prime}\right\|: z, z^{\prime} \in[\mathcal{B}, l]\right\}>0 .
$$

Now we check $\left(\Phi_{5}\right)$. Given a compact interval $I \subset(0, \infty)$ with $d:=\max I$ we set $l=[d / \theta]$ and $\mathcal{A}=[\mathcal{F}, l]$. We have $P^{+}[\mathcal{F}, l]=\left[P^{+} \mathcal{F}, l\right]$. Thus from (5.2)

$$
\inf \left\{\left\|z_{1}^{+}-z_{2}^{+}\right\|: z_{1}, z_{2} \in \mathcal{A}, z_{1}^{+} \neq z_{2}^{+}\right\}>0 .
$$

In addition, $\mathcal{A}$ is a $(C)_{I}$-attractor by Lemma 4.4 and $\mathcal{A}$ is bounded because $\|z\| \leq l \max \{\|\bar{z}\|: \bar{z} \in \mathcal{F}\}$ for all $z \in \mathcal{A}$. Therefore, by Theorem $5.2, \Phi$ has a unbounded sequence of critical values which contradicts with the assumption $(*)$, and hence $\Phi$ has infinite many geometrically distinct nontrivial critical points. Thus our multiplicity results follows. 


\section{Acknowledgements}

Many thanks to the referee for careful reading and helpful suggestions which make us possible to refine the paper.

\section{References}

[1] N. Ackermann, On a periodic Schrödinger equation with nonlinear superlinear part, Math. Z. 248 (2004), 423-443.

[2] N. Ackermann, A superposition principle and multibump solutions of periodic Schrödinger equations, J. Func. Anal. 234 (2) (2006), 277-320.

[3] C. O. Alves, P. C. Carrião, O. H. Miyagaki, On the existence of positive solutions of a perturbed Hamiltonian system in $\mathbb{R}^{N}$, J. Math. Anal. Appl. 276 (2002), 673-690.

[4] A. I. Ávila, J. Yang, Multiple solutions of nonlinear elliptic systems, Nonlinear Diff. Eqns. Appl. 12 (2005), 459-479.

[5] A. I. Ávila, J. Yang, On the existence and shape of least energy solutions for some elliptic systems, J. Diff. Eqns. 191 (2003), 348-376.

[6] T. Bartsch, D. G. de Figueiredo, Infinitely many solutions of nonlinear elliptic systems, Progress in Nonlinear Differential Equations and Their Applications, Vol. 35, Birkhäuser, Basel/Switzerland, 1999, 51-67.

[7] T. Bartsch, Y. Ding, Deformation theorems on non-metrizable vector spaces and applications to critical point theory, Math. Nach. 279 (2006), 1-22.

[8] V. Benci, P. H. Rabinowitz, Critical point theorems for indefinite functionals, Inven. Math. 52 (1979), 241-273.

[9] V. Coti Zelati, P. H. Rabinowitz, Homoclinic orbits for second order Hamiltonian systems possessing superquadratic potentials, J. Amer. Math. Soc. 4 (1991), 693 727.

[10] V. Coti Zelati, P. H. Rabinowitz, Homoclinic type solutions for a semilinear elliptic PDE on $\mathbb{R}^{N}$, Comm. Pure Appl. Math. 45 (1992), 1217-1269.

[11] D. G. de Figueiredo, Y. Ding, Strongly indefinite functionals and multiple solutions of elliptic systems, Tran. Amer. Math. Soc. 355 (2003), 2973-2989.

[12] D. G. de Figueiredo, P. L. Felmer, On superquadratic elliptic systems, Tran. Amer. Math. Soc. 343 (1994), 97-116.

[13] D. G. de Figueiredo, J. M. Do ó, B. Ruf, An Orlicz-space approach to superlinear elliptic systems, J. Func. Anal. 224 (2005), 471-496.

[14] D. G. de Figueiredo, J. Yang, Decay, symmetry and existence of solutions of semilinear elliptic systems, Nonlinear Anal. 33 (1998), 211-234.

[15] Y. Ding, L. Jeanjean, Homoclinic orbits for a non periodic Hamiltonian system, J. Differential Equations 237 (2007), 473-490.

[16] J. Hulshof, R. C. A. M. van de Vorst, Differential systems with strongly variational structure, J. Func. Anal. 114 (1993), 32-58.

[17] L. Jeanjean, On the existence of bounded Palais-Smale sequences and application to a Landesmann-Lazer type problem set on $\mathbb{R}^{N}$, Proc. Roy. Soc Edinburgh 129 A (1999), 787-809. 
[18] W. Kryszewski, A. Szulkin, An infinite dimensional Morse theory with applications, Tran. Amer. Math. Soc. 349 (1997), 3181-3234.

[19] W. Kryszewski, A. Szulkin, Generalized linking theorem with an application to semilinear Schrödinger equations, Adv. Differential Equations 3 (1998), 441-472.

[20] G. Li, A. Szulkin, An asymptotically periodic Schrödinger equation with indefinite linear part, Comm. Contemp. Math. 4 (2002), 763-776.

[21] G. Li, J. Yang, Asymptotically linear elliptic systems, Comm. Partial Diff. Eqns. 29 (2004), 925-954.

[22] A. Pistoia, M. Ramos, Locating the peaks of the least energy solutions to an elliptic system with Neumann boundary conditions, J. Diff. Eqns. 201 (2004), 160-176.

[23] M. Reed, B. Simon, Methods of Modern Mathematical Physics, IV Analysis of Operators, Academic Press, New York, 1978.

[24] E. Séré, Existence of infinitely many homoclinic orbits in Hamiltonian systems, Math. Z. 209 (1992), 133-160.

[25] B. Sirakov, On the existence of solutions of Hamiltonian elliptic systems in $R^{N}, A d v$. Diff. Eqns. 5 (2000), 1445-1464.

[26] C. Troestler, M. Willem, Nontrivial solution of a semilinear Schrödinger equation, Comm. Partial Diff. Eqns. 21 (1996), 1431-1449.

[27] M. Willem, Minimax Theorems, Birkhäuser, Berlin, 1996

[28] J. Yang, Nontrivial solutions of semilinear elliptic systems in $\mathbb{R}^{N}$, Electron. J. Diff. Eqns. 6 (2001), 343-357.

Fukun Zhao

Department of Mathematics

Yunnan Normal University

Kunming 650092 Yunnan

P.R. China

and

Institute of Mathematics

AMSS, CAS

Beijing 100080

P.R.China

e-mail: fukunzhao@163.com

Leiga Zhao and Yanheng Ding

Institute of Mathematics

AMSS, CAS

Beijing 100080

P.R.China

e-mail: zhaolg@amss.ac.cn

dingyh@math.ac.cn

Received: 20 December 2007.

Revised: 12 May 2008.

Accepted: 20 May 2008. 